\title{
Isomorphisms on Weighed Banach Spaces of Harmonic and Holomorphic Functions
}

\author{
Enrique Jordá and Ana María Zarco \\ Departamento de Matemática Aplicada, E. Politécnica Superior de Alcoy, Universidad Politécnica de Valencia, \\ Plaza Ferrándiz y Carbonell 2, 03801 Alcoy, Spain
}

Correspondence should be addressed to Enrique Jordá; ejorda@mat.upv.es

Received 30 May 2013; Accepted 3 August 2013

Academic Editor: Miguel Martin

Copyright (C) 2013 E. Jordá and A. M. Zarco. This is an open access article distributed under the Creative Commons Attribution License, which permits unrestricted use, distribution, and reproduction in any medium, provided the original work is properly cited.

For an arbitrary open subset $U \subset \mathbb{R}^{d}$ or $U \subseteq \mathbb{C}^{d}$ and a continuous function $\left.v: U \rightarrow\right] 0, \infty\left[\right.$ we show that the space $h_{v_{0}}(U)$ of weighed harmonic functions is almost isometric to a (closed) subspace of $c_{0}$, thus extending a theorem due to Bonet and Wolf for spaces of holomorphic functions $H_{v_{0}}(U)$ on open sets $U \subset \mathbb{C}^{d}$. Inspired by recent work of Boyd and Rueda, we characterize in terms of the extremal points of the dual of $h_{v_{0}}(U)$ when $h_{v_{0}}(U)$ is isometric to a subspace of $c_{0}$. Some geometric conditions on an open set $U \subseteq \mathbb{C}^{d}$ and convexity conditions on a weight $v$ on $U$ are given to ensure that neither $H_{v_{0}}(U)$ nor $h_{v_{0}}(U)$ are rotund.

\section{Introduction, Notation, and Preliminaries}

Let $U \subseteq \mathbb{R}^{d}$ or $U \subseteq \mathbb{C}^{d}$ be a nonempty open set. A weight on $U$ is a function $v: U \rightarrow \mathbb{R}$ which is strictly positive, continuous, and bounded. Let $h(U)$ denote the space of complex valued harmonic functions on $U$. For a weight $v$ the weighed Banach spaces of harmonic functions with weight $v$ are defined by

$$
\begin{gathered}
h_{v}(U):=\left\{f \in h(U):\|f\|_{v}:=\sup _{z \in G} v(z)|f(z)|<\infty\right\}, \\
h_{v_{0}}(U):=\{f \in h(U): v f \text { vanishes at infinity on } U\} .
\end{gathered}
$$

A function $g: U \rightarrow \mathbb{C}$ is said to vanish at infinity on $U$ if for each $\varepsilon>0$ there exists a compact subset $K \subset U$ such that $|g(x)| \leq \varepsilon$ for each $x \in U \backslash K$.

If $U \subseteq \mathbb{C}^{d}$ then the weighed Banach spaces of holomorphic functions $H_{v}(U)$ and $H_{v_{0}}(U)$ are defined in the same way. The unit disc and the unit circle in $\mathbb{C}$ are denoted by $\mathbb{D}$ and $\Gamma$, respectively. $U$ is said to be balanced if $\lambda z \in U$ for each $z \in U$ and each $\lambda \in \mathbb{D}$. A weight $v$ on a balanced open subset $U \subseteq \mathbb{C}^{n}$ is said to be radial whenever $v(\lambda z)=v(z)$ for each $\lambda \in \Gamma$.
Weighed Banach spaces of holomorphic functions with weighed supremum norms and composition operators between them have been studied by Bierstedt et al., Bonet et al., Contreras and Hernández-Díaz, García et al., Montes Rodríguez, and others; see [1-8] and the references therein.

Spaces of harmonic functions have been investigated by Shields and Williams [9], in connection with the growth of the harmonic conjugate of a function. In [10], they proved results of duality for weighed spaces of harmonic functions on the open unit disk. Lusky also considers weighed spaces of harmonic functions in $[11,12]$, where the isomorphism classes in the case of radial weights on the disk are determined. In [13] the authors have studied associated weights in weighed Banach spaces of harmonic functions and composition operators with holomorphic symbol between weighed spaces of pluriharmonic functions.

In [14-16] Boyd and Rueda investigate the geometry of $h_{v_{0}}(U)$ and $H v_{0}(U)$ where $U$ is an open set of the complex plane. Moreover they examined the extreme points of $h_{v_{0}}(U)^{*}$ and of $H_{v_{0}}(U)^{*}$ for $U \subseteq \mathbb{C}^{d}$.

In this work we prove in the second section that, for any weight $v$ on an open subset $U$ of $\mathbb{R}^{d}$, the weighed Banach space $h_{v_{0}}(U)$ of harmonic functions with $v f$ vanishing at infinity on $U$ is always isomorphic to a subspace of $c_{0}$ thus 
extending a result due to Bonet and Wolf [17] for holomorphic functions. Further, given $0<\varepsilon<1$ one can get a linear mapping $T: h_{v_{0}}(U) \rightarrow c_{0}$ such that $(1-\varepsilon)\|f\|_{v} \leq$ $\|T(f)\| \leq\|f\|_{v}$ for each $f \in h_{v_{0}}(U)$; that is, $h_{v_{0}}(U)$ is almost isometric to a subspace of $c_{0}$. We show that for radial weights in balanced open subsets the isomorphism cannot be isometric, generalizing [14, Theorem 16, Corollary 17] to spaces of harmonic functions defined on not necessarily bounded sets. We also show in this section that if the unit ball of $h_{v_{0}}(U)^{*}$ has a big quantity of extremal points, then $h_{v_{0}}(U)$ is not isometric to any subspace of $c_{0}$.

In the third section we mainly focus ourselves on weighed spaces of holomorphic and harmonic functions defined on balanced subsets $U \subseteq \mathbb{C}^{d}$ and with radial weights. We study the extremal points of the unit sphere of $H_{v_{0}}(U)^{*}$ in the way that Boyd and Rueda initiated in $[14,15]$. More precisely, we give conditions on $U$ and $v$ under which one can find in the unit sphere of $H_{v_{0}}(U)^{*}$ a big quantity of extremal points. We show that these conditions imply that $H_{v_{0}}(U)$ is not rotund.

Our notation for functional analysis and Banach space theory is standard. We refer to [18]. For a Banach space $X$ we denote by $B_{X}$ and $S_{X}$ its unit ball and its unit sphere, respectively. A (real or complex) Banach space $X$ is said to be rotund whenever $\|(x+y) / 2\|<1$ for $\|x\|=\|y\|=1$ and $x \neq y$. A vector $x$ in the unit sphere $S_{X}$ of a Banach space $X$ is called extremal if there are not $y, z \in S_{X}$ such that $y \neq z$ and $x=(1 / 2)(y+z)$, and it is called exposed if there is $x^{*} \in X^{*}$ such that $\left\|x^{*}\right\|=1, x^{*}(x)=1$, and $x^{*}(y) \neq 1$ for each $y \in S_{X} \backslash\{x\}$. If $X$ is complex this last condition is equivalent to $x^{*}(x)=1$ and $\operatorname{Re} x^{*}(y)<1$ for $y \in B_{X} \backslash\{x\}$, where $\operatorname{Re} z$ denotes the real part of a complex number $z$. If $x$ is exposed, then $x$ is also extremal. If $X$ is a dual Banach space and the functional exposing $x$ can be taken in the predual, then $x$ is called weak ${ }^{*}$ exposed. For $x, y \in \mathbb{R}^{d}$ (or $\mathbb{C}^{d}$ ) we denote by $\langle x, y\rangle$ its canonical scalar product. The Euclidean norm is denoted by $\|\cdot\|$. When we consider other norms on a finite dimensional space they are denoted by $|\cdot|$, making an abuse of notation because the modulus of a complex number is denoted also by $|\cdot|$.

For a Hausdorff locally compact topological space $Q$ we denote by $\widehat{Q}=\{Q \cup \infty\}$ its Alexandroff compactification. The boundary of a subset $U$ of a topological space is denoted by $\partial U$. If $U \subseteq \mathbb{R}^{d}$ is an open subset then the space $h_{v_{0}}(U)$ is formed by the harmonic functions on $U$ such that $v f^{0}$ can be extended continuously to $\widehat{U}$ by $(v f)(\infty)=0$. For an open set $U \subseteq \mathbb{R}^{d}$, the isometry between $h_{v_{0}}(U)$ and a subspace of $C(\widehat{U})$ by means of $f \mapsto v f$ permits to use the same argument as in $\left[14\right.$, Proposition 1] to show that the extreme points of $h_{v_{0}}(U)^{*}$ are contained in the set

$$
\left\{\lambda v(z) \delta_{z}: \lambda \in \Gamma, z \in U\right\},
$$

where $\delta_{z}$ denotes the evaluation at $z$. The following definitions are due to Boyd and Rueda [14-16]. The harmonic $v$ boundary is defined as follows:

$$
b_{v}(U):=\left\{z \in U: v(z) \delta_{z}\right.
$$

is extremal in the unit sphere of $\left.h_{v_{0}}(U)^{*}\right\}$.
The set of harmonic $v$-peak points is defined as

$$
p_{v}(U):=\left\{z \in U: v(z) \delta_{z}\right.
$$

is weak ${ }^{*}$ exposed in the unit sphere of $\left.h_{v_{0}}(U)^{*}\right\}$.

Remark 1. The same argument as in [15, Theorem 6] shows that to check that a functional of the form $v\left(x_{0}\right) \delta_{x_{0}}$ is weak* exposed it is enough to show that there exists $f$ in the unit ball of $h_{v_{0}}(U)$ such that $v\left(x_{0}\right) f\left(x_{0}\right)=1, v(x) \operatorname{Re} f(x)<1$ for each $x \in U \backslash\left\{x_{0}\right\}$.

If $U \subseteq \mathbb{C}^{d}$ then the holomorphic $v$-boundary and the set of holomorphic $v$-peak points are defined analogously, and they are denoted by $B_{v}(U)$ and $P_{v}(U)$, respectively. Since $H_{v_{0}}(U) \subset h_{v_{0}}(U)$, in this case, we have

$$
P_{v}(U) \subseteq B_{v}(U) \subseteq U,
$$

and also

$$
P_{v}(U) \subseteq p_{v}(U) \subseteq b_{v}(U) \subseteq U .
$$

We finish this section with this lemma also due to Boyd and Rueda.

Lemma 2 (see $[14,15])$. Let $U \subseteq \mathbb{R}^{d}\left(U \subseteq \mathbb{C}^{d}\right)$ be a nonempty open set, and let $v$ be a weight which vanishes at infinity on $U$.

(a) For any $\lambda \in \Gamma$ and $z \in U$ the functional $\lambda v(z) \delta_{z}$ is extremal in the unit sphere of $h_{v_{0}}(U)^{*}$ (is extremal in the unit sphere of $\left.H_{v_{0}}(U)^{*}\right)$ if and only if $z \in b_{v}(U)(z \in$ $\left.B_{v}(U)\right)$.

(b) If $U \subseteq \mathbb{C}^{d}$ is balanced, $v$ is radial and $\lambda \in \Gamma$ then $\lambda z \in$ $b_{v}(U)$ for each $z \in b_{v}(U)\left(\lambda z \in B_{v}(U)\right.$ for each $z \in$ $\left.B_{v}(U)\right)$.

\section{Isomorphisms on $h_{v_{0}}(U)$}

We extend below the main theorem of [17] to weighed spaces of harmonic functions. The proof is analogous to the proof given by Bonet and Wolf. One only has to take in account that there are Cauchy type inequalities which are valid for the derivatives of harmonic functions $[19,2.4]$. We include it for the sake of completeness. The argument was inspired by one used by Kalton and Werner [20, Corollary 4.9] to show that the little Bloch space $B_{0}$ embeds almost isometrically in $c_{0}$. Lusky showed in [21] that whenever $v$ is a radial weight on $\mathbb{D}$, then $h_{v_{0}}(\mathbb{D})$ is isomorphic to a subspace of $c_{0}$.

Theorem 3. Let $U \subset \mathbb{R}^{d}$ be a nonempty open set, let $v$ be a weight on $U$, and let $\varepsilon>0$. There exists an isomorphism $T$ : $h_{v_{0}}(U) \rightarrow c_{0}$ such that $(1-\varepsilon)\|f\|_{v} \leq\|T(f)\| \leq\|f\|_{v}$ for each $f^{0} \in h_{v_{0}}(U)$.

Proof. Let $\left(K_{j}\right)_{j}$ be a fundamental sequence of compact subsets of $U$, and let $\varepsilon>0$. We claim that there exists a sequence of pairwise disjoint finite subsets $\left(F_{j}\right)_{j}$ such that 
$F_{j} \subset A_{j}:=K_{j} \backslash K_{j-1}^{\circ}$ and satisfying that for each $f \in h_{v_{0}}$ with $\|f\|_{v}=1$

$$
\sup _{x \in A_{j}} v(x)|f(x)| \leq \varepsilon+\sup _{y \in F_{j}} v(y)|f(y)| .
$$

This implies that if we denote $F:=\cup_{j} F_{j}$, then we have

$$
\sup _{x \in F} v(x)|f(x)| \leq\|f\|_{v} \leq(1+\varepsilon) \sup _{x \in F} v(x)|f(x)|,
$$

and consequently, if we write $F$ as a sequence $\left(w_{j}\right)_{j}$, then the linear mapping $T: h_{v_{0}}(U) \rightarrow c_{0}, f \mapsto\left(v\left(w_{j}\right) f\left(w_{j}\right)\right)_{j}$ is injective; it has closed range and norm no greater than one, and its inverse $T^{-1}$ defined on a closed subspace of $c_{0}$ has norm less than or equal to $1+\varepsilon$. Since this is true for each $\varepsilon>0$, this is equivalent to our statement.

We proceed to show the claim. For each $j \in \mathbb{N}$ we define

$$
M_{j}:=\sup _{x \in K_{j}} v(x), \quad a_{j}:=\min \left(\frac{1}{2} d\left(K_{j}, \mathbb{R}^{N} \backslash K_{j+1}^{\circ}\right), 1\right) \text {, }
$$

and we select $x^{(j)} \in K_{j}$ such that $v\left(x^{(j)}\right)=\min _{x \in K_{j}} v(x)$. For each $f \in h_{v_{0}}(U)$ with $\|f\|_{v}=1$ and each $j \in \mathbb{N}$, we have

$$
\sup _{x \in K_{j}}|f(x)| \leq \frac{1}{v\left(x^{(j)}\right)}
$$

If $x \in K_{j}$ and $\zeta \in D\left(x, a_{j}\right)$ (here $D\left(x, a_{j}\right)$ denotes the closed ball with center $x$ and radius $\left.a_{j}\right)$, then from the definition of $a_{j}$ we deduce that $\zeta \in K_{j+1}$, and hence, together with the previous inequality,

$$
|f(\zeta)| \leq \frac{1}{v\left(x^{(j+1)}\right)}
$$

By Cauchy's inequalities for harmonic functions given in [19, 2.4 ], there exists a positive number $C$ (independent of $x$ ) such that, for each $\zeta \in D\left(x, a_{j}\right)$, the $i$ th derivative of $f$ satisfies

$$
\left|D_{i} f(\zeta)\right| \leq \frac{C}{a_{j} v\left(x^{(j+1)}\right)}, \quad i=1,2, \ldots, d
$$

By compactness we can get a finite subset $F_{j}$ of $A_{j}$ such that

$$
A_{j} \subset \bigcup_{x \in F_{j}}\left\{x^{\prime} \in U:\left|x^{\prime}-x\right|<\delta_{j},\left|v\left(x^{\prime}\right)-v(x)\right|<\delta_{j}\right\},
$$

where $\delta_{j}$ has been chosen satisfying $\delta_{j}<a_{j}$ and

$$
\left(\frac{1}{v\left(x^{(j)}\right)}+\frac{M_{j+1} d C}{a_{j} v\left(x^{(j+1)}\right)}\right) \delta_{j}<\varepsilon
$$

Consequently, for each $x \in A_{j}$, there exists $w \in F_{j}$ with $|w-x|<\delta_{j}$ and $|v(w)-v(x)|<\delta_{j}$. On the other hand,
$D\left(x, \delta_{j}\right) \subset D\left(x, a_{j}\right) \subset K_{j+1}$ for each $x=\left(x_{1}, x_{2}, \ldots, x_{d}\right) \epsilon$ $A_{j}$. We write $w=\left(w_{1}, w_{2}, \ldots, w_{d}\right)$ to get

$$
\begin{aligned}
|f(x)|= & \left|f\left(x_{1}, \ldots, x_{d}\right)\right| \\
\leq & \left|f\left(x_{1}, \ldots, x_{d}\right)-f\left(w_{1}, x_{2}, \ldots, x_{d}\right)\right| \\
& +\left|f\left(w_{1}, x_{2}, \ldots, x_{d}\right)-f\left(w_{1}, w_{2}, \ldots, x_{d}\right)\right| \\
& +\cdots+\left|f\left(w_{1}, w_{2}, \ldots, w_{d}\right)\right| .
\end{aligned}
$$

We apply the mean value theorem to each difference and (12) to get

$$
\begin{aligned}
|f(x)| & \leq \frac{C}{a_{j} v\left(x^{(j+1)}\right)} d|x-w|+|f(w)| \\
& \leq \frac{d \delta_{j} C}{a_{j} v\left(x^{(j+1)}\right)}+|f(w)| .
\end{aligned}
$$

Now, since $w \in K_{j+1}$,

$$
\begin{aligned}
v(x)|f(x)| & \leq|v(x)-v(w)||f(x)|+v(w)|f(x)| \\
& \leq \delta_{j} \frac{1}{v\left(x^{(j)}\right)}+v(w)\left(\frac{d \delta_{j} C}{a_{j} v\left(x^{(j+1)}\right)}+|f(w)|\right) \\
& \leq \frac{\delta_{j}}{v\left(x^{(j)}\right)}+M_{j+1} \frac{d \delta_{j} C}{a_{j} v\left(x^{(j+1)}\right)}+v(w)|f(w)| \\
& \leq \varepsilon+v(w)|f(w)| \leq \varepsilon+\max _{w \in F_{j}} v(w)|f(w)| .
\end{aligned}
$$

Therefore,

$$
\sup _{x \in A_{j}} v(x)|f(x)| \leq \varepsilon+\max _{w \in F_{j}} v(w)|f(w)| .
$$

Again as in [17], since each infinite dimensional subspace of $c_{0}$ contains a complemented subspace isomorphic to $c_{0}$, the following result follows.

Corollary 4. There are not infinite dimensional subspaces of $h_{v_{0}}(U)$ which are reflexive.

In order to get examples where the isomorphism above cannot be an isometry, we will consider a condition which is inspired by the results of Boyd and Rueda [14, Theorem 16, Corollary 17] for weighed spaces defined on bounded open sets of $\mathbb{C}^{d}$. First we observe that for any weight $v$ for which $h_{v_{0}}(U)$ contains the polynomials it happens that the functionals $\left\{\delta_{z}: z \in U\right\} \subseteq h_{v_{0}}(U)^{*}$ are linearly independent. If $U$ is bounded this is the case for any weight $v$ which tends to zero at the boundary. If $U=\mathbb{R}^{d}$, then the condition is satisfied when $v=g(|x|)$, with $|\cdot|$ being a norm in $\mathbb{R}^{d}$ and $g:[0, \infty[\rightarrow] 0, \infty$ [ being a rapidly decreasing continuous function, that is, with $\lim _{t \rightarrow \infty} t^{n} g(t)=0$ for any $n \in \mathbb{N}$. First we extend easily [14, Lemma 10] to unbounded domains. 
Lemma 5. Let $U \subset \mathbb{R}^{d}$ be a nonempty open set, and let $v$ be a continuous strictly positive weight which vanishes at infinity on $U$. If $h_{v_{0}}(U)$ contains the polynomials, then the map $\mu: U \rightarrow$ $\left(h_{v_{0}}(U)^{*}, w^{*}\right)$, and $z \mapsto v(z) \delta_{z}$ is a homeomorphism onto its image.

Proof. Since the polynomials belong to $h_{v_{0}}(U)$, we get that $\left\{\delta_{z}: z \in U\right\} \subset h_{v_{0}}(U)^{*}$ are linearly independent functionals. We consider the map $\widehat{\mu}: \widehat{U} \rightarrow\left(h_{v_{0}}(U)^{*}, w^{*}\right), z \rightarrow v(z) \delta_{z}$ if $z \in U$ and $\widehat{\mu}(\infty)=0$. The very definition of $h_{v_{0}}(U)$ implies that $\widehat{\mu}$ is continuous, and it is injective by the linear independence of the evaluations. By the compactness of $\widehat{U}$ it follows that $\widehat{\mu}$ is a homeomorphism, and hence also its restriction $\mu$ to $U$.

Proposition 6. Let $U \subset \mathbb{R}^{d}\left(U \subset \mathbb{C}^{d}\right)$ be a nonempty open set, and let $v$ be a weight for which $h_{v_{0}}(U)\left(H_{v_{0}}(U)\right)$ contains the polynomials. Assume that $b_{v}(U)\left(B_{v}(U)\right)$ is not discrete. Then $h_{v_{0}}(U)\left(H_{v_{0}}(U)\right)$ cannot be isometric to any subspace of $c_{0}$.

Proof. We give only the proof for the harmonic case. Denote by $\widehat{\mathbb{N}}=\mathbb{N} \cup\{\infty\}$ the Alexandroff compactification of $\mathbb{N}$. Let $T: h_{v_{0}}(U) \rightarrow c_{0}$ be an isometry onto its image. Let $F:=T\left(h_{v_{0}}(U)\right) \subseteq c_{0}$. Since $c_{0}$ is the subspace of $C(\widehat{\mathbb{N}})$ formed by the functions which vanish at $\infty$, the extremal points of the unit sphere of $F^{*}$ are contained in $\left\{\lambda \delta_{n}: \lambda \in \Gamma, n \in\right.$ $\mathbb{N}\}$ (cf. [22, Lemma V.8.6]). Let $Q: F \rightarrow h_{v_{0}}(U)$ be the inverse isometry of $T$. The transpose linear mapping $Q^{t}$ : $h_{v_{0}}(U)^{*} \rightarrow F^{*}$ is also an isometry, and it maps extremal points to extremal points. Thus for each $z \in b_{v}(U)$ there exists $\lambda_{z} \in \Gamma$ and $n(z) \in \mathbb{N}$ such that $Q^{t}\left(v(z) \delta_{z}\right)=\lambda_{z} \delta_{n(z)}$. From the linear independence of $\left\{\delta_{z}: z \in U\right\}$ in $h_{v_{0}}^{*}$, we conclude that $n(z) \neq n(w)$ for $z, w \in U$ with $z \neq w$, and then $b_{v}(U)$ is countable and $\left\{\lambda_{z} \delta_{n(z)}: z \in b_{v}(U)\right\}$ is discrete for the weak ${ }^{*}$ topology, since it is a sequence in the sphere of $F^{*}$ which is $\sigma\left(F^{*}, F\right)$ convergent to 0 . But $Q^{t}$ is a weak ${ }^{*}$ weak ${ }^{*}$ homeomorphism. Hence we conclude from Lemma 5 that $b_{v}(U)$ is discrete.

Now we apply the set of extremal points of the unit ball of a dual Banach space which is never empty and Lemma 2 to get the following result.

Corollary 7. If $U \subseteq \mathbb{C}^{d}$ is a balanced open set and $v$ is a radial weight which vanishes at infinity on $U$, then neither $H_{v_{0}}(U)$ nor $h_{v_{0}}(U)$ is isometric to a subspace of $c_{0}$.

Although we do not have an example of weight $v$ on $U$ such that $b_{v}(U)$ is a discrete subset of $U$, we see below that we can reformulate the problem of finding an example of space $h_{v_{0}}(U)$ which can be isometrically embedded in $c_{0}$ in terms of the possible existence of an open set $U \subseteq \mathbb{R}^{d}$ and a weight $v$ such that $b_{v}(U)$ is not discrete. Of course the analogue result is also true for the holomorphic case. In view of Lemma 2 this is not possible for radial weights in balanced domains.
Proposition 8. If $U \subseteq \mathbb{R}^{d}$ is an open set and $v$ is a weight on $U$ such that $h_{v_{0}}(U)$ contains the polynomials and $b_{v}(U)$ is discrete, then $h_{v_{0}}(U)$ embeds isometrically in $c_{0}$.

Proof. Since the closed unit ball $D$ of $h_{v_{0}}(U)^{*}$ is the weak ${ }^{*}$ closure of the absolutely convex hull of the extremal points of the sphere, Lemma 2(a) implies that $D$ is in fact the closure of the absolutely convex hull of $\left\{v(z) \delta_{z}: z \in U\right\}$. Thus this implies that $b_{v}(U)$ cannot be finite, and since it is discrete we have that $b_{v}(U)=\left(x_{n}\right)_{n}$ with $x_{n}$ tending to infinity on $U$ when $n$ goes to infinity. We get now that $D$ is the weak ${ }^{*}$ closure of the absolutely convex hull of $\left\{v\left(x_{n}\right) \delta_{x_{n}}: n \in \mathbb{N}\right\}$, and hence, for each $f \in h_{v_{0}}(U)$, it holds:

$$
\|f\|_{v}:=\sup _{n \in \mathbb{N}} v\left(x_{n}\right)\left|f\left(x_{n}\right)\right| .
$$

Since $x_{n}$ tends to infinity on $U$, we have that the linear map

$$
T: h_{v_{0}}(U) \longrightarrow c_{0}, \quad f \longmapsto\left(v\left(x_{n}\right) f\left(x_{n}\right)\right)_{n}
$$

is an isometry.

\section{Geometry of Weighed Banach Spaces of Holomorphic and Harmonic Functions and Their Duals}

In [15, Theorem 29] Boyd and Rueda showed that if $U \subseteq$ $\mathbb{C}^{d}$ is a balanced bounded open set and $v$ is a radial weight vanishing at infinity on $U$ such that $P_{v}(U)=B_{v}(U)$ then $H_{v_{0}}(U)$ is not rotund and then neither $h_{v_{0}}(U)$ is. This condition is trivially satisfied when $P_{v}(U)=U$. To the best of our knowledge there are not so far concrete examples of open subsets $U \subset \mathbb{C}^{d}$ and weights $v$ on $U$ such that $B_{v}(U) \backslash$ $P_{v}(U) \neq \emptyset$. In the concrete examples of spaces $H_{v_{0}}(U)$ given in [15] where $B_{v}(U)$ is calculated the equality $B_{v}(U)=P_{v}(U)$ is always satisfied. With a similar proof to the one of [15, Theorem 29], we present below a new condition for spaces $H_{v_{0}}(U)$ with $U$ balanced and $v$ radial depending only on the size of $P_{v}(U)$ which ensures that $H_{v_{0}}(U)$ is not rotund.

Proposition 9. Let $U \subseteq \mathbb{C}^{d}$ be the open unit ball for a norm $|\cdot|$ in $\mathbb{C}^{d}$, and let $v=g(|x|)$ for $g:[0,1] \rightarrow[0, \infty[$ be a nonincreasing continuous function with $g(1)=0$. If there exists $z_{0} \in \partial U$ such that the set $\left\{t z_{0}: 0<t<1\right\} \subseteq P_{v}(U)$, then $H_{v_{0}}(U)$ is not rotund and then neither $h_{v_{0}}(U)$ is.

Proof. Let $\varphi$ be a linear map on $\mathbb{C}^{d}$ such that $\left\|\varphi^{2}\right\|_{v}=1$. Since $v \varphi^{2}$ is continuous on $\widehat{U}$ and vanishes at infinity, there exists $z_{1} \in U, z_{1} \neq 0$ such that

$$
1=\left\|\varphi^{2}\right\|_{v}=v\left(z_{1}\right) \varphi^{2}\left(z_{1}\right)=v\left(-z_{1}\right) \varphi^{2}\left(-z_{1}\right) .
$$

Since $\left|z_{0}\right|=1$, we apply the theorem of Hahn-Banach to get $y \in \mathbb{C}^{d}$ such that $\left\langle z_{0}, y\right\rangle=1$ and $|\langle z, y\rangle| \leq|z|$ for each $z \epsilon$ $\mathbb{C}^{d}$. Let $r_{0}:=\left|z_{1}\right|$. We define

$$
h: U \longrightarrow \mathbb{C}, \quad z \longrightarrow \varphi^{2}\left(\frac{\langle z, y\rangle}{r_{0}} z_{1}\right) .
$$


We have

$$
\begin{aligned}
v\left(r_{0} z_{0}\right) h\left(r_{0} z_{0}\right) & =g\left(r_{0}\right) \varphi^{2}\left(z_{1}\right) \\
& =g\left(\left|z_{1}\right|\right) \varphi^{2}\left(z_{1}\right)=v\left(z_{1}\right) \varphi^{2}\left(z_{1}\right)=1 .
\end{aligned}
$$

Since $\varphi$ is linear we also get

$$
v\left(-r_{0} z_{0}\right) h\left(-r_{0} z_{0}\right)=g\left(r_{0}\right) \varphi^{2}\left(-z_{1}\right)=v\left(-z_{1}\right) \varphi^{2}\left(-z_{1}\right)=1 .
$$

For $z \in U$ arbitrary, we use that $\left|z_{1} / r_{0}\right|=1,|\langle z, y\rangle| \leq|z|$, and $g$ is not increasing to compute

$$
\begin{aligned}
v(z)|h(z)| & =g(|z|)|h(z)| \leq g(|\langle z, y\rangle|)|h(z)| \\
& =v\left(\frac{\langle z, y\rangle}{r_{0}} z_{1}\right)\left|\varphi^{2}\left(\frac{\langle z, y\rangle}{r_{0}} z_{1}\right)\right| \leq\left\|\varphi^{2}\right\|_{v}=1 .
\end{aligned}
$$

Thus $h$ is in the unit ball of $H_{v_{0}}(U)$ and

$$
v\left(r_{0} z_{0}\right) \delta_{r_{0} z_{0}}(h)=v\left(-r_{0} z_{0}\right) \delta_{\left(-r_{0} z_{0}\right)}(h)=1 .
$$

Since $r_{0} z_{0}$ is assumed to be a peak point we can find $f$ in the unit ball of $H_{v_{0}}(U)$ different from $h$ such that $v\left(r_{0} z_{0}\right) f\left(r_{0} z_{0}\right)=$ 1 and $v(z) \operatorname{Re}(f(z))<1$ for each $z \in U \backslash\left\{r_{0} z_{0}\right\}$. Hence we conclude from

$$
\left\|\frac{f+h}{2}\right\|_{v}=v\left(r_{0} z_{0}\right) \frac{\left(f\left(r_{0} z_{0}\right)+h\left(r_{0} z_{0}\right)\right)}{2}=1
$$

that $H_{v_{0}}(U)$ is not rotund.

Let $g:[0,1] \rightarrow[0, \infty[$ be a function such that $g(1)=$ $0, g$ is decreasing, $g$ is twice differentiable in $] 0,1[$, and $\log (1 / g(t))$ is convex. In [15, Corollary 12] the authors showed that for a weight $v$ defined in the Euclidean unit ball $U$ of $\mathbb{C}^{d}$ by $v(x)=g(\|x\|)$ the equality $P_{v}(U)=U$ holds, and then $H_{v_{0}}(U)$ cannot be rotund by [15, Theorem 29]. Also if $U_{1} \subseteq \mathbb{C}^{d}$ and $U_{2} \subseteq \mathbb{C}^{k}$ are the Euclidean unit balls and $v_{1}$ and $v_{2}$ satisfy this last condition for certain $g_{1}$ and $g_{2}$, then $\left.v: U_{1} \times U_{2} \rightarrow\right] 0, \infty\left[, v(x, y)=v_{1}(x) v_{2}(y)\right.$ also satisfies $P_{v}(U)=U$ (cf. [15, Proposition 26]). We present below new examples of spaces $H_{v_{0}}(U)$ which cannot be rotund. In the required assumptions, besides considering a wider class of open sets, we remove from the weight the condition of differentiability given by Boyd and Rueda as we did in [13, Theorem 1] to give conditions under which $v=\widetilde{v}, \widetilde{v}$ being the associated weight introduced by Bierstedt et al. in [23]. The same technique permits us to get concrete radial weights $v(x)=g(\|x\|)$ defined in the Euclidean ball $U$ of $\mathbb{R}^{d}$ with $d$ odd such that the spaces $h_{v_{0}}(U)$ are not isometric to any subspace of $c_{0}$. If $d=2 k$, then the assertion is a consequence of Corollary 7 since $U$ is the Euclidean unit ball of $\mathbb{C}^{k}$ and $H_{v_{0}}(U) \subset h_{v_{0}}(U)$.

Proposition 10. Let $g:[0,1] \rightarrow[0, \infty[$ be a continuous decreasing function such that $g(1)=0$ and $\log (1 / g)$ is strictly convex in $[0,1[$. (a) Let $U \subseteq \mathbb{C}^{d}$ be the unit ball for a norm $|\cdot|$ and $v: U \rightarrow$ ]0, $\infty\left[, x \mapsto g(|x|)\right.$. Then $H_{v_{0}}(U)$ is not rotund (and then neither $h_{v_{0}}(U)$ is).

(b) Let $U \subseteq \mathbb{R}^{d}$ be the Euclidean unit ball and $v(x)=$ $g(\|x\|)$ for $x \in U$. Then $h_{v_{0}}(U)$ is not isometric to any subspace of $c_{0}$.

Proof. Let $h(r):=\log (1 / g(r)), 0 \leq r<1$. Since $h$ is increasing and strictly convex, for each $0 \leq r_{0}<1$, the map

$$
\left[0,1\left[\backslash\left\{r_{0}\right\} \longrightarrow\right] 0, \infty\left[, \quad r \longmapsto \frac{\left(h(r)-h\left(r_{0}\right)\right)}{\left(r-r_{0}\right)}\right.\right.
$$

is increasing. Hence for each $0 \leq r_{0}<1$ there exists $\alpha_{0} \geq 0$ which depends on $r_{0}$ such that

$$
h(r)-h\left(r_{0}\right)>\alpha_{0}\left(r-r_{0}\right)
$$

for all $r \in\left[0,1\left[\backslash\left\{r_{0}\right\}\right.\right.$. Now we compute

$$
\begin{aligned}
\sup _{0 \leq r<1, r \neq r_{0}} g(r) \exp \left(\alpha_{0} r\right)= & \exp \left(\sup _{0<r<1, r \neq r_{0}}\left(-h(r)+\alpha_{0} r\right)\right) \\
& <\exp \left(\operatorname { s u p } _ { 0 \leq r < 1 , r \neq r _ { 0 } } \left(-\alpha_{0}\left(r-r_{0}\right)\right.\right. \\
& \left.\left.\quad-h\left(r_{0}\right)+\alpha_{0} r\right)\right) \\
& =g\left(r_{0}\right) \exp \left(\alpha_{0} r_{0}\right) .
\end{aligned}
$$

We prove (a). Straszewicz's theorem [24, Theorem 7.89] together with the Krein-Milman theorem imply that there exists $z_{0} \in \partial U$ which is exposed. Let $y \in \mathbb{C}^{d}$ such that $\left\langle z_{0}, y\right\rangle=1$ and $\operatorname{Re}(\langle z, y\rangle)<|z|$ if $z \in U \backslash\left\{r z_{0}: 0 \leq r<1\right\}$. Let $0 \leq r_{0}<1$ be arbitrary, and let $\alpha_{0}$ be as above. The function $f: U \rightarrow \mathbb{C}$ defined by $f_{0}(z):=\left(\exp \left(\alpha_{0}\langle z, y\rangle\right)\right) /$ $\left(g\left(r_{0}\right) \exp \left(\alpha_{0} r_{0}\right)\right)$ is holomorphic and $v\left(r_{0} z_{0}\right) f_{0}\left(r_{0} z_{0}\right)=1$. It follows immediately from (30) that if $0 \leq r<1, r \neq r_{0}$

$$
v\left(r z_{0}\right)\left|f_{0}\left(r z_{0}\right)\right|=\frac{g(r) \exp \left(\alpha_{0} r\right)}{g\left(r_{0}\right) \exp \left(\alpha_{0} r_{0}\right)}<1 .
$$

If $z \in U \backslash\left\{r z_{0}: 0 \leq r<1\right\}$, then we use (30) and $\operatorname{Re}(\langle z, y\rangle)<|z|$ to get

$$
\begin{aligned}
v(z)\left|f_{0}(z)\right| & =g(|z|) \frac{\exp \left(\alpha_{0} \operatorname{Re}(\langle z, y\rangle)\right)}{g\left(r_{0}\right) \exp \left(\alpha_{0} r_{0}\right)} \\
& <g(|z|) \frac{\exp \left(\alpha_{0}|z|\right)}{g\left(r_{0}\right) \exp \left(\alpha_{0} r_{0}\right)} \leq 1 .
\end{aligned}
$$

Hence $f_{0}$ peaks $v\left(r_{0} z_{0}\right) \delta_{r_{0} z_{0}}$. We conclude that $\left\{r z_{0}: 0 \leq r<\right.$ $1\} \subseteq P_{v}(U)$ since $r_{0}$ is arbitrary. We apply Proposition 9 to get that $H_{v_{0}}(U)$ is not rotund.

To prove (b) we consider $x_{0} \in \mathbb{R}^{d}$ with $0<\left\|x_{0}\right\|=r_{0}<1$ and get $\alpha_{0}$ again as above. Let $A$ be an orthogonal transformation such that $A\left(x_{0}\right)=\left(r_{0}, 0, \ldots, 0\right)$. For $x=\left(x_{1}, \ldots, x_{d}\right)$ 
we take the harmonic function $h(x):=\exp \left(\alpha_{0} x_{1}\right) \cos \left(\alpha_{0} x_{2}\right)$ and we define $f_{0}(x):=h \circ A(x)$ for $x \in U$, which is harmonic; see [19, Chapter 1]. A similar argument as in (a) permits us to conclude that $v\left(x_{0}\right) f_{0}\left(x_{0}\right)=1$ and $v(x)|f(x)|<1$ for each $x \in U \backslash\left\{x_{0}\right\}$. Hence $p_{v}(U)=U$, and therefore $h_{v_{0}}(U)$ is not isometrically embedded in $c_{0}$ by Proposition 6 .

Remark 11. (a) An inspection of the proofs of Propositions 9 and 10 shows that the statements remain true when $U=\mathbb{C}^{d}$ and $g:[0, \infty[\rightarrow] 0, \infty[$ is a decreasing continuous function which satisfies that $\log (1 / g)$ is strictly convex and $g(t)=$ $o\left(e^{-a t}\right)$ when $t$ goes to infinity for each $a>0$.

(b) For any increasing function $h:[0,1[\rightarrow[0, \infty[$ which is strictly convex and satisfies $\lim _{t \rightarrow 1} h(t)=\infty$ (or $h$ : $\left[0, \infty[\rightarrow] 0, \infty\left[\right.\right.$ with $e^{a t}=o\left(e^{h(t)}\right)$ when $t$ goes to infinity for each $a>0$ ), the function $g(t)=e^{-h(t)}$ satisfies the hypothesis of Propositions 9 and 10. The examples given in [15, Example 13] of weights in the Euclidean unit ball can be obtained using this general method.

\section{Acknowledgments}

The authors are grateful to J. Bonet for a lot of ideas and discussions during all this work. They are also indebted to P. Rueda, who besides giving them some references provided them some unpublished work which has inspired a big part of this paper. They also thank M. Maestre for his careful reading of the paper and helpful discussions. Finally, they thank the referees for the careful analysis of the paper and the important suggestions which they gave us. The research of the first author was supported by MICINN andFEDER, Project MTM2010-15200. The research of both authors is partially supported by Programa de Apoyo a la Investigación y Desarrollo de la UPV PAID-06-12.

\section{References}

[1] K. D. Bierstedt, J. Bonet, and A. Galbis, "Weighted spaces of holomorphic functions on balanced domains," The Michigan Mathematical Journal, vol. 40, no. 2, pp. 271-297, 1993.

[2] J. Bonet, P. Domański, and M. Lindström, "Essential norm and weak compactness of composition operators on weighted Banach spaces of analytic functions," Canadian Mathematical Bulletin, vol. 42, no. 2, pp. 139-148, 1999.

[3] J. Bonet, P. Domański, and M. Lindström, "Weakly compact composition operators on analytic vector-valued function spaces," Annales Academioe Scientiarum Fennico. Mathematica, vol. 26, no. 1, pp. 233-248, 2001.

[4] J. Bonet, P. Domański, M. Lindström, and J. Taskinen, "Composition operators between weighted Banach spaces of analytic functions," Journal of the Australian Mathematical Society. Series A, vol. 64, no. 1, pp. 101-118, 1998.

[5] M. D. Contreras and A. G. Hernandez-Diaz, "Weighted composition operators in weighted Banach spaces of analytic functions," Journal of the Australian Mathematical Society. Series A, vol. 69, no. 1, pp. 41-60, 2000.

[6] D. García, M. Maestre, and P. Rueda, "Weighted spaces of holomorphic functions on Banach spaces," Studia Mathematica, vol. 138, no. 1, pp. 1-24, 2000.
[7] D. García, M. Maestre, and P. Sevilla-Peris, "Composition operators between weighted spaces of holomorphic functions on Banach spaces," Annales Academice Scientiarum Fennicce. Mathematica, vol. 29, no. 1, pp. 81-98, 2004.

[8] A. Montes-Rodríguez, "Weighted composition operators on weighted Banach spaces of analytic functions," Journal of the London Mathematical Society, vol. 61, no. 3, pp. 872-884, 2000.

[9] A. L. Shields and D. L. Williams, "Bounded projections and the growth of harmonic conjugates in the unit disc," The Michigan Mathematical Journal, vol. 29, no. 1, pp. 3-25, 1982.

[10] A. L. Shields and D. L. Williams, "Bounded projections, duality, and multipliers in spaces of harmonic functions," Journal für die Reine und Angewandte Mathematik, vol. 299-300, pp. 256-279, 1978.

[11] W. Lusky, "On weighted spaces of harmonic and holomorphic functions," Journal of the London Mathematical Society, vol. 51, no. 2, pp. 309-320, 1995.

[12] W. Lusky, "On the isomorphism classes of weighted spaces of harmonic and holomorphic functions," Studia Mathematica, vol. 175, no. 1, pp. 19-45, 2006.

[13] E. Jordá and A. M. Zarco, "Weighted Banach spaces of harmonic functions," Revista de la Real Academia de Ciencias Exactas, Fisicas y Naturales. Serie A, 2012.

[14] C. Boyd and P. Rueda, "The -boundary of weighted spaces of holomorphic functions," Annales Academice Scientiarum Fennica. Mathematica, vol. 30, no. 2, pp. 337-352, 2005.

[15] C. Boyd and P. Rueda, "Complete weights and -peak points of spaces of weighted holomorphic functions," Israel Journal of Mathematics, vol. 155, pp. 57-80, 2006.

[16] C. Boyd and P. Rueda, "Isometries of weighted spaces of harmonic functions," Potential Analysis, vol. 29, no. 1, pp. 37-48, 2008.

[17] J. Bonet and E. Wolf, "A note on weighted Banach spaces of holomorphic functions," Archiv der Mathematik, vol. 81, no. 6, pp. 650-654, 2003.

[18] M. Fabian, P. Habala, P. Hájek, V. Montesinos, and V. Zizler, Banach Space Theory, CMS Books in Mathematics/Ouvrages de Mathématiques de la SMC, Springer, New York, NY, USA, 2011.

[19] S. Axler, P. Bourdon, and W. Ramey, Harmonic Function Theory, vol. 137 of Graduate Texts in Mathematics, Springer, New York, NY, USA, 2nd edition, 2001.

[20] N. J. Kalton and D. Werner, "Property $(M), M$-ideals, and almost isometric structure of Banach spaces," Journal für die Reine und Angewandte Mathematik, vol. 461, pp. 137-178, 1995.

[21] W. Lusky, "On the structure of $H_{v_{0}}(D)$ and $h_{v_{0}}(D)$," Mathematische Nachrichten, vol. 159, pp. 279-289, 1992.

[22] N. Dunford and J. T. Schwartz, Linear Operators. Part I:Wiley Classics Library, Wiley Classics Library, John Wiley \& Sons, New York, NY, USA, 1988.

[23] K. D. Bierstedt, J. Bonet, and J. Taskinen, "Associated weights and spaces of holomorphic functions," Studia Mathematica, vol. 127, no. 2, pp. 137-168, 1998.

[24] C. D. Aliprantis and K. C. Border, Infinite Dimensional Analysis: A Hitchhiker's Guide, Springer, Berlin, Germany, 3rd edition, 2006. 


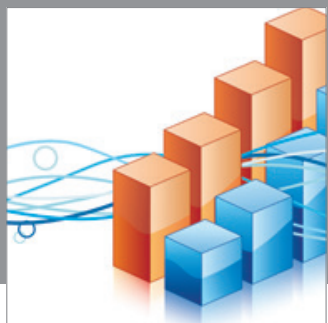

Advances in

Operations Research

mansans

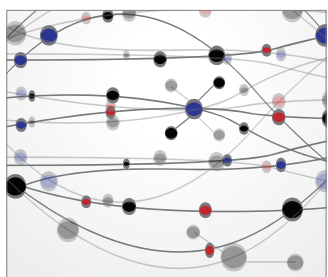

The Scientific World Journal
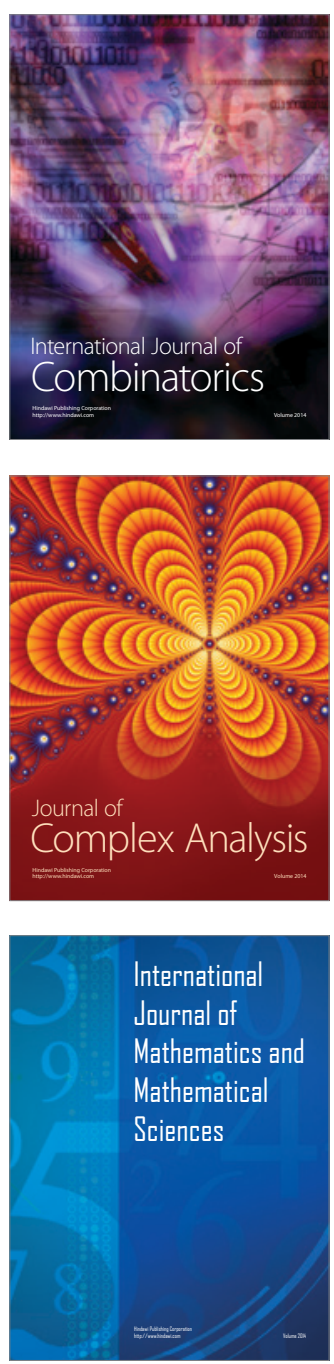
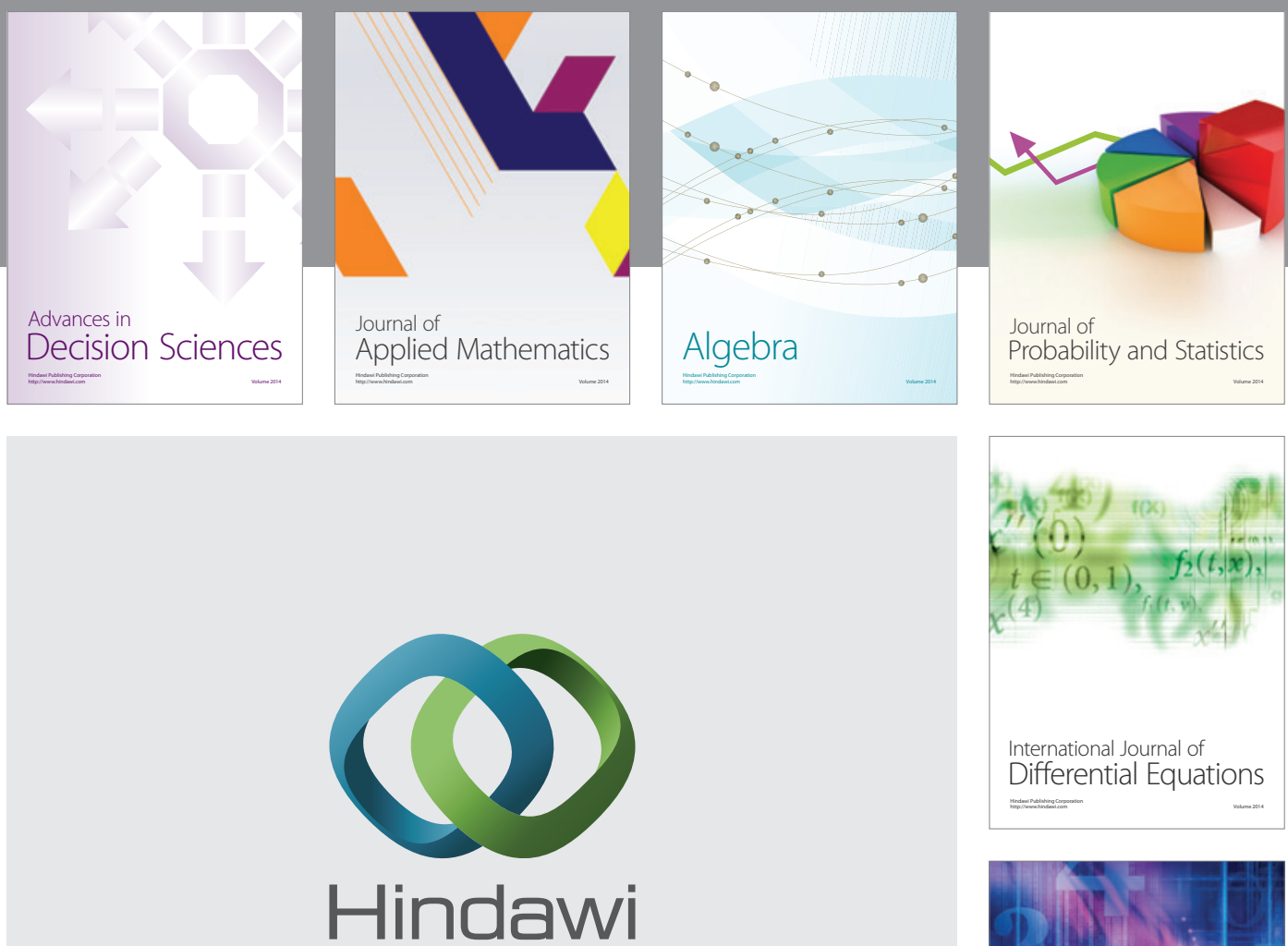

Submit your manuscripts at http://www.hindawi.com
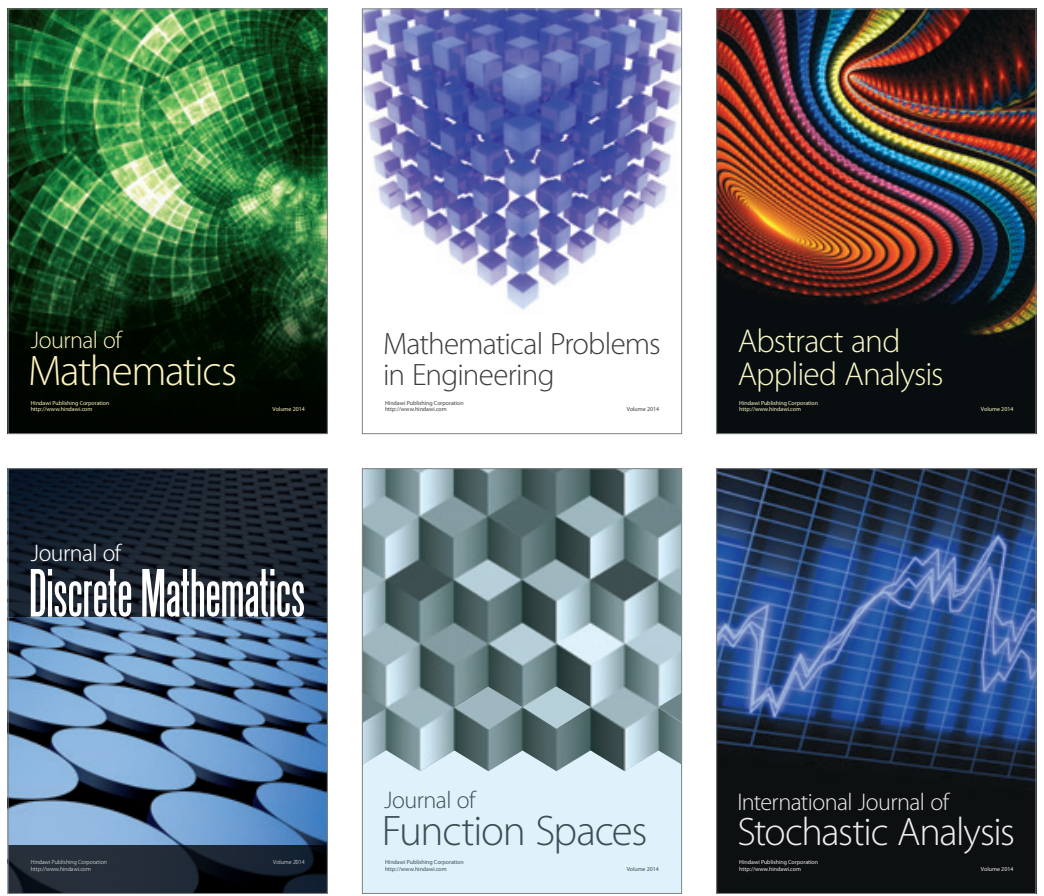

Journal of

Function Spaces

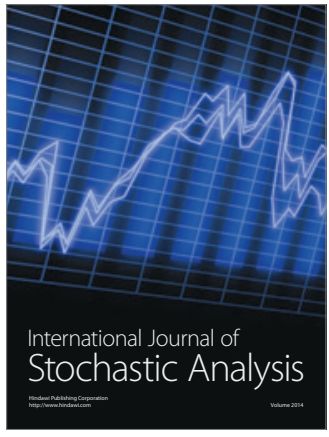

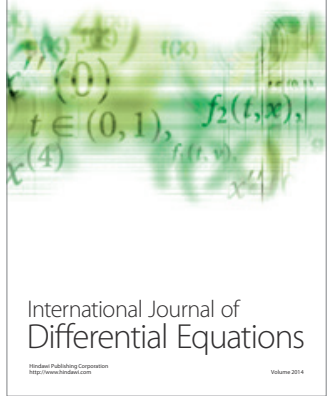
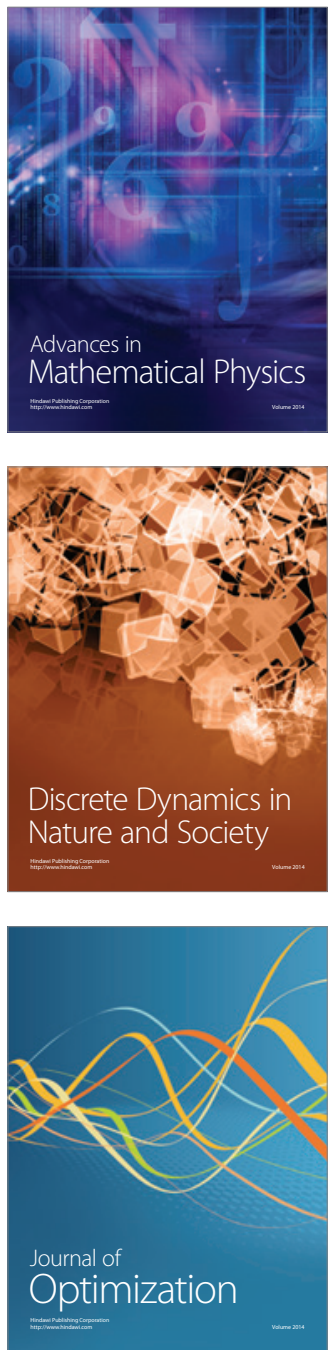